\title{
Subpellicular and flagellar microtubules of Trypanosoma brucei are
}

\section{extensively glutamylated}

\author{
André Schneider ${ }^{1}$, Uwe Plessmann² and Klaus Weber,* \\ ${ }^{1}$ University of Fribourg, Institute of Zoology, Pérolles, $\mathrm{CH} 1700$ Fribourg, Switzerland \\ 2Max Planck Institute for Biophysical Chemistry, Department of Biochemistry, PO Box 2841, D-37018, Goettingen, Germany \\ *Author for correspondence
}

\section{SUMMARY}

To determine the spectrum of tubulin variants in cytoskeletons of Trypanosoma brucei carboxy-terminal fragments of $\alpha$ - and $\beta$-tubulin were isolated and characterized by sequencing and mass spectrometry. All variants arise by posttranslational modifications. We confirm the presence of tyrosinated and detyrosinated $\alpha$-tubulin. Unexpectedly, but in line with its sequence, $\beta$-tubulin also occurs with and without its carboxy-terminal tyrosine. Both tyrosinated and detyrosinated $\alpha$ - and $\beta$-tubulins are extensively glutamylated. Unglutamylated tubulins are only trace components of the cytoskeletal microtubules. The maximal numbers of glutamyl residues in the lateral chain are $\mathbf{1 5}$ and 6 for $\alpha$ - and $\beta$-tubulin, respectively. The oligoglutamyl side chain is linked via an isopeptide bond to glutamic acid residues 445 of $\alpha$ - and 435 of $\beta$-tubulin. The same sites are used in glutamylated tubulins of mammalian brain. No tubulin variants based on polyglycylation are detected in cytoskeletal preparations or in isolated flagella. Tubulin specific incorporation of radioactive glutamate but not of glycine is observed when protein biosynthesis is completely inhibited in Trypanosoma cells. Possible reasons for the absence of polyglycylated tubulins from the trypanosomal axoneme are discussed. Finally we show that lysine 40 of the flagellar $\alpha$-tubulin is completely acetylated.

Key words: Acetylation, Carboxypeptidase, Polyglutamylation, Posttranslational modification, Tyrosination

\section{INTRODUCTION}

Microtubules are involved in eukaryotic cell division, directed intracellular transport, the movement of cilia and flagella and they also influence the dynamic organisation of cellular morphology. The $\alpha \beta$-tubulin heterodimer, the structural unit of the microtubules, is the target of a number of posttranslational modifications (PTMs). These PTMs fall into two categories. Some, such as the acetylation of lysine 40 in certain $\alpha$-tubulins (LeDizet and Piperno, 1987) and the phosphorylation sites in the carboxy-terminal region (see for instance Alexander et al., 1991; Rüdiger and Weber, 1993) are more general PTMs observed also in other proteins. Other PTMs seem to be tubulin-specific and occur in the acidic carboxy-terminal region. The terminal tyrosine of certain $\alpha$-tubulins participates in a detyrosination/tyrosination cycle, which involves a carboxypeptidase-like activity and the well characterized tubulintyrosine ligase (Raybin and Flavin, 1975; Ersfeld et al., 1993; Thompson, 1982), which restores the tyrosine in an ATPdependent reaction. Once $\alpha$-tubulins have lost also the penultimate glutamic acid residue they are no longer a substrate for the ligase (Paturle et al., 1989; Paturle-Lafanechère et al., 1991; Rüdiger et al., 1994). Two other PTMs unique to certain tubulins were established by mass spectrometry of the carboxyterminal peptides. They involve a polyglutamyl or polyglycyl sidechain of variable length attached via an isopeptide bond to the $\gamma$-carboxylate of a particular glutamic acid residue of $\alpha$ - and $\beta$-tubulin. In the case of polyglutamylation the position of this modified residue has been established for all brain tubulins (Eddé et al., 1990; Alexander et al., 1991; Redeker et al., 1992; Rüdiger et al., 1992; Mary et al., 1994). While the other tubulin-specific PTMs first emerged in animal cells and tissues polyglycination was first documented in the ciliary axonemal microtubules of the ciliate Paramecium (Redeker et al., 1994). It is also present in bull sperm flagella (Rüdiger et al., 1995a) and echinoderm sperm axonemes (Mary et al., 1996; Multigner et al., 1996) and has recently been found in the flagellated diplomonad Giardia lamblia (Weber et al., 1996), one of the oldest eukaryotes (Sogin et al., 1989).

Trypanosoma are members of the Kinetoplastida, which are considered to be more primitive and older eukaryotes than the ciliates (Sogin et al., 1989; Cavalier-Smith, 1993). They have a remarkably simple cytoskeleton which, unlike in other cells is based primarily on one filament system, the microtubules. Trypanosomal microtubules are found in two major organizations: a subpellicular cage of singlet microtubules, which are in close contact with the cell membrane, and the flagellar axoneme. This unique organization and the fact that tubulins are the most abundant proteins in these cells has attracted some interest in T. brucei as a model system to study a prototype microtubular cytoskeleton. The trypanosomal cytoskeleton has been characterized in considerable detail on the ultrastructural, the biochemical as well as on the genetic level (for review see Seebeck et al., 1990). 
Trypanosomes were the first protozoan organisms for which a functional detyrosination/tyrosination cycle of $\alpha$-tubulin was documented by labelling experiments performed under conditions in which protein synthesis was inhibited (Stieger et al., 1984). Similar experiments showed the presence of $\alpha$-tubulin acetylation (Schneider et al., 1987). Here we used protein chemistry and mass spectrometry of the carboxy-terminal tubulin peptides together with in vivo labelling experiments to analyse whether glutamylation and glycylation is already present in $T$. brucei, one of the earliest known eukaryotes.

\section{MATERIALS AND METHODS}

\section{Cells}

Procyclic T. brucei, stock 427, were grown at $27^{\circ} \mathrm{C}$ in SDM-79 medium supplemented with $5 \%$ fetal bovine serum. Cells were harvested at late log phase corresponding to $1.5 \times 10^{7}$ to $2.5 \times 10^{7}$ cells $/ \mathrm{ml}$.

\section{Cytoskeletal and flagellar fractions}

Detergent extraction of total cytoskeleton and isolation of flagella was as described (Schneider et al., 1987). From $2 \times 10^{9}$ cells approximately $2.5 \mathrm{mg}$ of cytoskeletal or $0.5 \mathrm{mg}$ of flagellar proteins were obtained. Both fractions were dissolved in SDS sample buffer, boiled and $2 \mathrm{mg}$ each was subjected to SDS-PAGE using a preparative $10 \%$ gel. After staining with Coomassie brilliant blue the major band corresponding to $\alpha$ - and $\beta$-tubulin was excised, washed with water and frozen at $-70^{\circ} \mathrm{C}$ until use.

\section{Isolation and characterization of carboxy-terminal fragments}

The $\alpha$-tubulin fragment was obtained by digestion with endoproteinase Lys $C$ and expected to provide residues 431 to 451 in the protein sequence predicted by Kimmel et al. (1985). Tubulin present in dye stained gel fragments was electrophoretically concentrated into a thin band by the agarose gel concentration system (Multigner et al., 1996; Rider et al., 1995). Lys C (Boehringer, Mannheim, Germany) was used at $3 \mu \mathrm{g} / \mathrm{ml}$ for 16 hours at $37^{\circ} \mathrm{C}$ in $0.1 \mathrm{M}$ Tris- $\mathrm{HCl}, \mathrm{pH} 8.5$, $5 \%$ in acetonitrile. The digest was recovered in $20 \mathrm{mM}$ Na-phosphate, pH 7.0 (buffer A) using a fast desalting column and the SMART system (Pharmacia, Uppsala, Sweden). It was separated on a Mono Q column $(1.6 \mathrm{~mm} \times 50 \mathrm{~mm})$, equilibrated in buffer A, with a $2.4 \mathrm{ml}$ salt gradient ( 0 to $0.5 \mathrm{M} \mathrm{NaCl}$ in buffer $\mathrm{A}$ ) followed by a $0.9 \mathrm{M}$ salt wash. The flow rate was $100 \mu \mathrm{l} /$ minute and fractions of $100 \mu \mathrm{l}$ were collected. The elution profile was monitored by absorption at $214 \mathrm{~nm}$. All later emerging peaks were subjected to reverse phase HPLC on a Vydac 218 TP51 column with a $2.4 \mathrm{ml}$ linear gradient (10 to $80 \%$ solvent B) at a flow rate of $40 \mu \mathrm{l} / \mathrm{minute}$. Solvents A and B were $0.1 \%$ trifluoroacetic acid (TFA) and 70\% acetonitrile in $0.08 \%$ TFA, respectively. Elution was monitored by absorption of $214 \mathrm{~nm}$ and peak fractions were collected manually.

The $\beta$-tubulin fragment was obtained by $\mathrm{CNBr}$ cleavage and expected to provide residues 416 to 442 in the protein sequence predicted by Kimmel et al. (1985). Stained gel fragments were processed for in situ CNBr treatment as described by Rüdiger et al. (1995a). The lyophilized fragments were dissolved in buffer A and separated by Mono Q chromatography and reverse phase HPLC as described above.

All peptide peaks from the HPLC profiles were characterized by mass spectrometry using a KRATOS MALDI 3 time of flight mass spectrometer (Shimadzu, Duisburg, Germany) with $\alpha$-cyano-4hydroxy-cinnamic acid in 30\% acetonitrile, $0.06 \%$ TFA as matrix. Spectra of negative ions were recorded in the linear mode. Calibration was with bovine insulin, $\alpha$-melanocyte stimulating hormone and

synthetic peptides. Major peptide peaks (see Results) were also analyzed by automated Edman degradation using instruments with online phenylthiohydantoin amino acid analysis.

\section{Identification of $\mathbf{N}$-acetyl lysine at position $\mathbf{4 0}$ of flagellar $\alpha$-tubulin}

Since lysine 40, the acetylation site of Chlamydomonas and mammalian brain $\alpha$-tubulins (LeDizet and Piperno, 1987; Eddé et al., 1990 ), is situated very early in the largest $\mathrm{CNBr}$ fragment it is easy to monitor this residue by Edman degradation (Rüdiger and Weber, 1993). The CNBr fragments of Trypanosoma flagellar $\alpha$-tubulin were separated by SDS-PAGE and electrophoretically blotted on to a poly(vinylidenedifluoride) membrane. The largest $\mathrm{CNBr}$ fragment corresponding to residues 37 to 203 (Kimmel et al., 1985) was subjected to automated sequencing, which resolves the phenylthiohydantoin derivatives of lysine and $\mathrm{N}$ - $\varepsilon$-acetyl-lysine (LeDizet and Piperno, 1987).

\section{In vivo labelling with radioactive amino acids}

Labelling conditions for $\left[{ }^{3} \mathrm{H}\right]$ glutamate and $\left[{ }^{3} \mathrm{H}\right]$ glycine were essentially as described for $\left[{ }^{3} \mathrm{H}\right]$ tyrosine (Stieger et al., 1984). For each labelling experiment, $2 \times 10^{8}$ exponentially growing $\left(0.5-1.0 \times 10^{7}\right.$ cells $/ \mathrm{ml}$ ) trypanosome cells were used. Cells were harvested, washed once and resuspended in 1/10 of the original volume of HHP-84 medium containing all amino acids except the one corresponding to the radioactive label (Seebeck and Kurath, 1985). Each of the three cultures was split in two aliquots and incubated at $27^{\circ} \mathrm{C}$ for 30 minutes without and in the presence of protein biosynthesis inhibitors (50 $\mu \mathrm{g} / \mathrm{ml}$ of cycloheximide, $25 \mu \mathrm{g} / \mathrm{ml}$ each of puromycin and chloramphenicol). Subsequently $25 \mu \mathrm{Ci}$ each of $\left[{ }^{3} \mathrm{H}\right]$ tyrosine (spec. act. 52 $\mathrm{Ci} / \mathrm{mmol}$ ), $\left[{ }^{3} \mathrm{H}\right]$ glutamate (spec. act. $41 \mathrm{Ci} / \mathrm{mmol}$ ), or $\left[{ }^{3} \mathrm{H}\right]$ glycine (spec. act. $51 \mathrm{Ci} / \mathrm{mmol}$ ) were added and incubation was continued for 2 hours. Cells of all six cultures were harvested, washed in HHP-84 medium and dissolved in $100 \mu \mathrm{l}$ SDS sample buffer. Samples were boiled and $50 \mu \mathrm{l}$ each (approximately $50 \mu \mathrm{g}$ of proteins) were analysed by SDS-PAGE on a $10 \%$ gel. Gels were fixed, incubated for 30 minutes in $1 \mathrm{M}$ sodium salicylate and processed for fluorography. Dried gels were exposed on preflashed X-ray film (X-omat AR film, Kodak SA) for 1 to 40 days depending on the amino acid. Radioactive amino acids were from Dupont NEN.

\section{RESULTS}

Cytoskeletal preparations of Trypanosoma obtained by Triton X-100 extraction are dominated by a tubulin band and the polypeptides of the paraflagellar rod (Fig. 1). Aliquots of the gel fragments of the tubulin band from preparative SDS-PAGE were used to isolate and characterize the carboxy-terminal fragments of $\alpha$ and $\beta$-tubulin (see Materials and Methods).

Fig. 1. SDS-PAGE analysis of trypanosomal cytoskeletal preparation. Trypanosoma brucei cells were extracted with $0.5 \%$ Triton X-100 and the insoluble residue was subjected to SDS-PAGE using a $10 \%$ gel. Note the strong tubulin band (arrow) at $55 \mathrm{kDa}$ and the band of the paraflagellar rod (PFR) at $70 \mathrm{kDa}$.

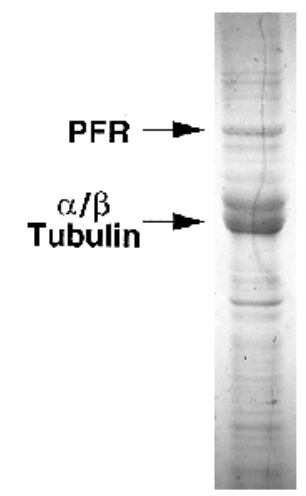




\section{$\alpha$-Tubulin variants of stable microtubules}

A tubulin digest with endoproteinase Lys $\mathrm{C}$ was first chromatographed on a small Mono Q column to isolate the acidic carboxy-terminal peptides. Later emerging peaks were subjected to further separation by reverse phase HPLC. All peak fractions from the HPLC experiments were monitored by mass spectrometry (Fig. 2) and three major fractions (marked in Table 1) were characterized by amino acid sequencing to verify the identity of the carboxy-terminal peptides versus the sequence predicted for $\alpha$-tubulin (Kimmel et al., 1985; see below). Table 1 summarizes the results. The $\alpha$ peptide occurred both in tyrosinated and detyrosinated form. The latter is about twice as abundant as the former. Due to glutamylation both peptides gave rise to numerous variants and the unglutamylated variants are only minor components (Table 1). The oligoglutamyl side chains varied in general between 1 and 4 but a minor fraction of the tyrosinated $\alpha$ carried up to 6 residues and a mod-
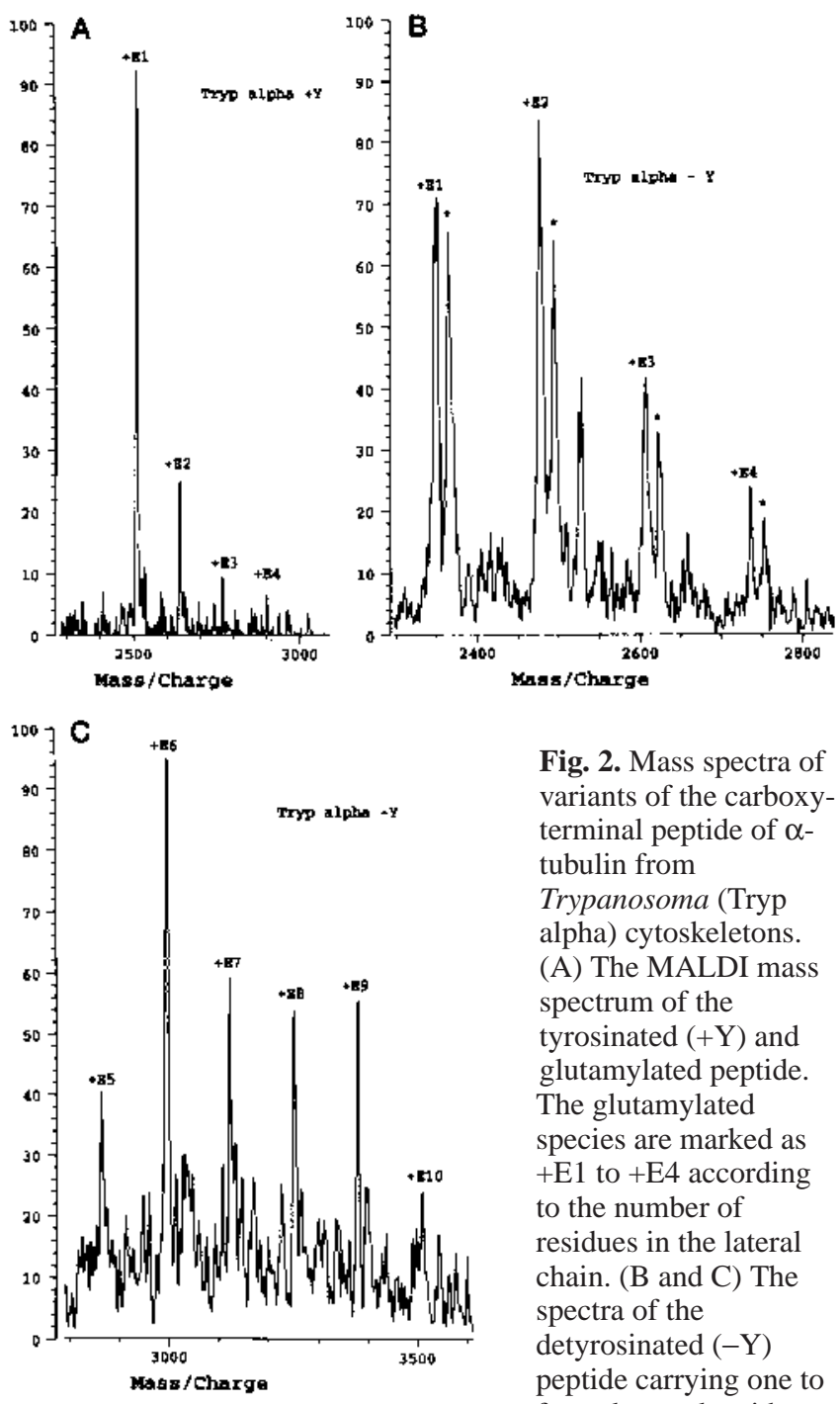

Fig. 2. Mass spectra of variants of the carboxyterminal peptide of $\alpha$ tubulin from

Trypanosoma (Tryp alpha) cytoskeletons. (A) The MALDI mass spectrum of the tyrosinated $(+\mathrm{Y})$ and glutamylated peptide. The glutamylated species are marked as $+\mathrm{E} 1$ to $+\mathrm{E} 4$ according to the number of residues in the lateral chain. (B and C) The spectra of the detyrosinated $(-\mathrm{Y})$ peptide carrying one to four glutamyl residues

$(+\mathrm{E} 1$ to $+\mathrm{E} 4$ in $\mathrm{B})$ or five to ten glutamyl residues $(+\mathrm{E} 5$ to $+\mathrm{E} 10$ in C) as side chain. Minor peaks marked by a star correspond to the fraction of the peptide in which the methionine was oxidized to methionine sulfoxide. (A,B,C) Three of the various HPLC fractions analyzed. For a summary see Table 1 . erately abundant fraction of detyrosinated $\alpha$ showed between 5 and 15 residues in the sidechain. Here the main components had lateral chains of 6 to 8 glutamyl residues (Table 1).

\section{Glutamylation of $\alpha$-tubulin involves glutamic acid 445}

Automated sequencing of three glutamylated variants (marked in Table 1) covered the carboxy-terminal 21 residues (positions 431 to 451 ) with or without the terminal tyrosine in the predicted Trypanosoma $\alpha$-tubulin sequence (Kimmel et al., 1985) except for position 445 where no phenylthiohydantoin derivative was obtained (Fig. 3). Since the sequence continued normally past this step the lateral oligoglutamyl chain must be linked to glutamic acid 445 via the isopeptide bond formed by the $\gamma$-carboxylate of this residue.

\section{$\beta$-tubulin variants of stable microtubules}

The sequence of Trypanosoma $\beta$-tubulin (Kimmel et al., 1985) predicts that endoproteinase Lys $\mathrm{C}$ should provide a carboxyterminal peptide of 50 residues (positions 393 to 442). Probably because of its size and/or solubility properties this peptide, like similar peptides from other $\beta$-tubulins (Rüdiger et al., 1995a; Weber et al., 1996), was not recovered. Therefore another aliquot of tubulin containing gel fragments was processed by in situ $\mathrm{CNBr}$ cleavage to isolate the predicted carboxy-terminal peptide of 27 residues (positions 416 to 442). Mono Q chromatography followed by HPLC and mass spectrometry identified a number of variants (Table 1). Unexpectedly, but in line with a carboxy-terminal tyrosine (Kimmel et al., 1985) the $\beta$ peptide occurred both with and without the terminal tyrosine (Figs 3 and 4).

Due to glutamylation both peptides gave rise to numerous variants and unglutamylated variants were not detected. The oligoglutamyl sidechains varied in length between 1 and 5 residues in the normal $\beta$ and 1 and 6 in the detyrosinated $\beta$ peptide. Table 1 summarizes the results. From a comparison of the different HPLC peaks and the species identified by mass spectrometry, we estimate that the sum of the tyrosinated variants exceeds the sum of the detyrosinated variants.

Automated sequencing of one glutamylated $\beta$ peptide (marked in Table 1) followed the predicted sequence (Kimmel

Table 1. Tubulin variants in Trypanosoma cytoskeletons

\begin{tabular}{|c|c|c|c|}
\hline Variant & $\begin{array}{c}\text { Glutamylation } \\
\text { level }\end{array}$ & $\begin{array}{c}\text { Main } \\
\text { components }\end{array}$ & $\begin{array}{c}\text { Relative } \\
\text { abundance }\end{array}$ \\
\hline$\alpha$ Tryrosinated & 0 & & Trace \\
\hline$\alpha$ Tryrosinated* & $1-4$ & 1 & Major \\
\hline$\alpha$ Tryrosinated & $4-6$ & 4 & Minor \\
\hline$\alpha$ Detyrosinated & 0 & & Minor \\
\hline$\alpha$ Detyrosinated* & $1-4$ & 2 & Major \\
\hline$\alpha$ Detyrosinated* & $5-15$ & $6,7,8$ & Moderate \\
\hline$\beta$ Normal & 0 & & Not found \\
\hline$\beta$ Normal & $1-5$ & 2 & Major \\
\hline$\beta$ Detyrosinated & 0 & & Not found \\
\hline$\beta$ Detyrosinated* & $1-6$ & 2 & Major \\
\hline
\end{tabular}

Tubulin variants identified via the mass spectra of their carboxy-terminal fragments. The glutamylation level refers to the number of residues per lateral chain and the most prominent number of residues are indicated. A rough estimate of the relative abundance was obtained by comparing the peak heights of the various HPLC chromatograms and the distribution of variants obtained by mass spectrometry. Peptides additionally characterized by automated sequencing are marked by a star. For sequences see Fig. 3. 


\section{Tryp. $\alpha \quad 431$ DYEEVGAESADMDGËEDVEY mam. CH 1 431 DYEEVGVDSVEGEGËEGEEY \\ Tryp. $\beta \quad 426$ QDAT IEEEGËFDEEOY mam. $\beta$ II 426 QDATADEQGËFEEEGEDEA}

Fig. 3. Identification of the carboxy-terminal peptides of $\alpha$ and $\beta$ trypanosomal tubulin and location of the polyglutamylation sites. Three glutamylated variants of the Trypanosoma (Tryp) $\alpha$ peptide, identified in Table 1, provided the sequence with and without the terminal tyrosine. This sequence fits the predicted residues 431 to 451 (Kimmel et al., 1985) except that at the position of glutamic acid 445 no phenylthiohydantoin derivative was obtained. Therefore Glu 445 carries the oligoglutamyl side chain via the isopeptide bond formed by its $\gamma$-carboxylate group. Note that mammalian brain (mam) $\alpha 1$ tubulin shows its glutamylation site also at glutamic acid 445 (Eddé et al., 1990; Rüdiger et al., 1992; Mary et al., 1994). Glutamylation sites are marked by an asterisk. One glutamylated variant of the Trypanosoma (Tryp) $\beta$ peptide (see Table 1) provided upon sequencing residues 416 to 437 of the carboxy-terminal peptide (416 to 442) predicted by Kimmel et al. (1995). Only positions 426 to 442 are shown. Since at position 435 no phenylthiohydantoin derivative was obtained Glu 435 carries the oligoglutamyl side chain (asterisk; for details see text). Note the carboxy-terminal tyrosine, which by mass spectrometry is absent from some $\beta$ peptide variants. Note also that mammalian brain (mam) $\beta$ II tubulin shows its glutamylation site on glutamic acid 435 (Redeker et al., 1992; Rüdiger et al., 1992; Mary et al., 1994).

et al., 1985) for positions 416 to 437 except that at position 435 no phenylthiohydantoin derivative was obtained. Since the sequence continued normally for two further steps glutamic acid 435 carries the lateral chain (Fig. 3). Because positions 438 to 442 were not reached by sequencing we cannot exclude the formal possibility of an additional modification site past residue 437.

\section{Posttranslational modifications of flagellar tubulins}

Using preparative SDS-PAGE on a preparation of purified flagella we collected the two tubulin bands separately rather than combining them as in the cytoskeletal preparation. Automated sequencing of a small aliquot showed that the two bands resembled pure $\alpha$ - and $\beta$-tubulin, respectively. Analysis of the carboxy-terminal peptides of flagellar tubulins showed no major differences versus the results obtained on cytoskeletal microtubules (Table 1) except that unglutamylated variants of $\alpha$-tubulin were not detected. Tubulins from both preparations lacked variants due to polyglycylation (see also below).

A further aliquot of the flagellar $\alpha$-tubulin was used to identify the lysine acetylation site previously predicted by Schneider et al. (1987). A CNBr digest was subjected to SDSPAGE and blotted on to a PVDF membrane. The large fragment of apparent molecular mass 19,000 was sequenced for 23 steps. The results cover positions 37 to 59 in the predicted sequence (Kimmel et al., 1985) except that at position 40 only $\mathrm{N}$-acetyl-lysine was detected. Thus lysine 40 is completely modified in the flagellar $\alpha$-tubulin.

\section{In vivo labelling experiments}

The presence of extensive glutamylation but at the same time the absence of glycylation in trypanosomal tubulins is surpris-
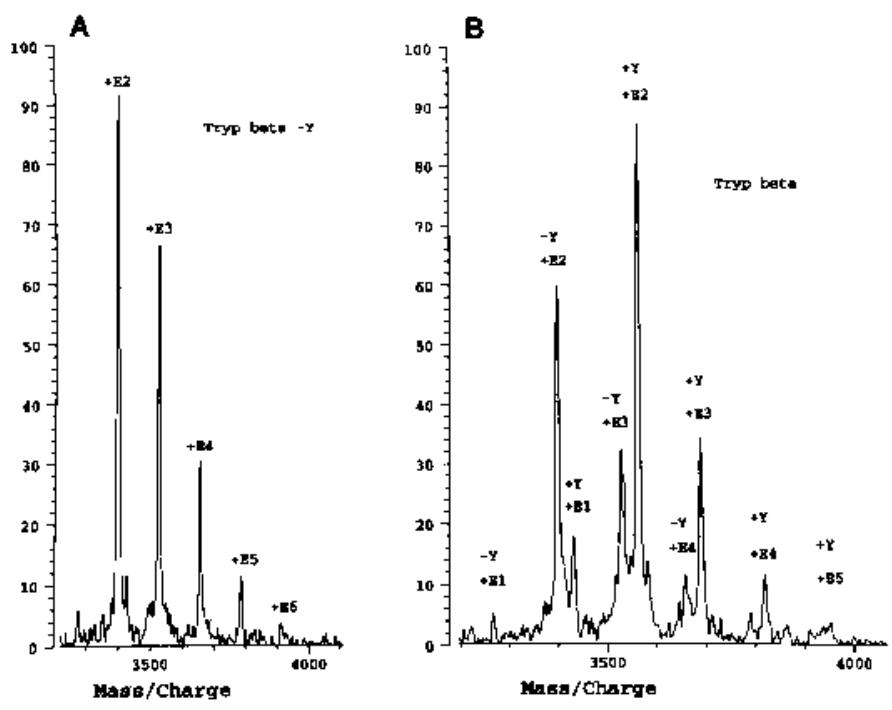

Fig. 4. Mass spectra of variants of the carboxy-terminal peptide of $\beta$ tubulin from Trypanosoma (Tryp beta) cytoskeletons. (A) The MALDI mass spectrum of the detyrosinated (-Y) and glutamylated $\beta$ peptide. The glutamylated species are marked as +E2 to +E6 according to the number of residues in the lateral chain. (B) The spectrum of detyrosinated $(-\mathrm{Y})$ and tyrosinated $(+\mathrm{Y})$ peptides carrying between one and five glutamyl residues indicated as $+\mathrm{E} 1$ to + E5. (A and B) Two of the various HPLC fractions analyzed. For a summary see Table 1 .

ing, since glycylated tubulin has been detected in Giardia lamblia, which in evolutionary terms is an even more primitive eukaryote than T. brucei (Weber et al., 1996). Therefore we tested whether radioactive glutamate and glycine can be incorporated into trypanosomal tubulin in vivo in the presence of protein synthesis inhibitors. Whereas translation is abolished under these conditions posttranslational modifications should not be affected. The same approach has previously been used with labelled tyrosine to demonstrate tubulin tyrosine ligase activity in T. brucei (Stieger et al., 1984). Trypanosomes were labelled with either $\left[{ }^{3} \mathrm{H}\right]$ tyrosine, $\left[{ }^{3} \mathrm{H}\right]$ glutamate or with $\left[{ }^{3} \mathrm{H}\right]$ glycine in the presence and absence of protein synthesis. Fig. 5 shows that in the absence of translational inhibitors all three amino acids are incorporated into total proteins, albeit at very different efficiencies. The incorporation level can be estimated by comparing fluorograms of different exposure times since the labelled amino acids were all of similar specific activity (see Materials and Methods). Tyrosine is incorporated into proteins approximately 10 -fold more efficiently than glycine, which is incorporated approximately 20-fold more efficiently than glutamate. The differences might be due to different intracellular pools of these amino acids. In the absence of translation $\left[{ }^{3} \mathrm{H}\right]$ tyrosine is incorporated into $\alpha$ tubulin (Fig. 5, lane 2) as expected (Stieger et al., 1984; Sherwin et al., 1987). A labelled band co-migrating with tyrosinated tubulin is also observed in the corresponding lane for $\left[{ }^{3} \mathrm{H}\right]$ glutamate (Fig. 5, lane 4). A comparison of the signal of the $\left[{ }^{3} \mathrm{H}\right]$ glutamate labelled tubulin band with the incorporation of $\left[{ }^{3} \mathrm{H}\right]$ glutamate into total proteins (Fig. 5, lane 3) indicates that glutamylation is very efficient. For glycine, however, no labelling is observed in the absence of translation. Since labelling of total proteins with $\left[{ }^{3} \mathrm{H}\right]$ glycine is more 


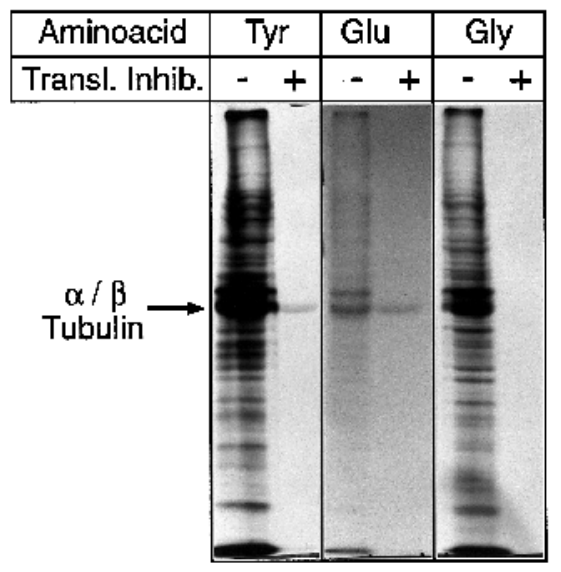

Fig 5. In vivo labelling of trypanosomal tubulins with $\left[{ }^{3} \mathrm{H}\right]$ tyrosine (Tyr), $\left[{ }^{3} \mathrm{H}\right]$ glutamate $(\mathrm{Glu})$ and $\left[{ }^{3} \mathrm{H}\right]$ glycine (Gly). Incorporation of $\left[{ }^{3} \mathrm{H}\right]$ amino acids into total protein marked by $(-)$. Incorporation of $\left[{ }^{3} \mathrm{H}\right]$ amino acids into total protein in the presence of inhibitors of protein biosynthesis marked by (+). All samples were resolved using the same 10\% SDS-gel. Lanes 1 and 2 were exposed for 16 hours, all other lanes for 20 days. Note the specific labelling of tubulin by tyrosine and glutamate in the presence of inhibitors of protein biosynthesis.

efficient than for $\left[{ }^{3} \mathrm{H}\right]$ glutamate (Fig. 5, lane 3 and 5) we conclude that tubulin glycylation does not occur in trypanosomes, or if it does, it must be at least 20-fold less efficient than tubulin glutamylation.

For the protein-chemical analysis we used trypanosomal cytoskeletons obtained by detergent extraction at $0^{\circ} \mathrm{C}$ in order to avoid proteolysis. Due to the low temperature we only isolate the stable microtubule populations i.e. the subpellicular and flagellar microtubules. Soluble tubulin and the labile spindle microtubules are not recovered. The in vivo labelling experiments, however, where total cellular extracts were analysed are not biased towards detecting stable microtubule populations exclusively. The combined protein-chemical results agree well with the in vivo labelling experiments. The inability to detect glycylated tubulin biochemically is therefore not due to the absence of unstable microtubules in the specimens analyzed.

\section{DISCUSSION}

The unique cytoskeleton of Trypanosoma is based on two sets of stable microtubules (Seebeck et al., 1988). There are about 100 helically arranged, subpellicular microtubules, which form a corset of parallel, interconnected microtubules in contact with the overlaying plasma membrane. The second microtubular organisation involves the typical $9+2$ structure of the axoneme in the single flagellum, which also displays a paraflagellar rod built from special coiled coil forming proteins (Schlaeppi et al., 1989). Previous studies on Trypanosoma under conditions where protein biosynthesis was completely inhibited showed that $\alpha$-tubulin selectively incorporates labelled acetate and tyrosine (Stieger et al., 1984; Schneider et al., 1987; Sherwin et al., 1987). Together with immunological results obtained with monoclonal antibodies of known epitope specificity this work established two posttranslational modifications, all specific for $\alpha$-tubulin. First, the carboxy-terminal tyrosine is subject to the detyrosination /tyrosination cycle based on a carboxypeptidase activity and the tubulin-tyrosine ligase, which restores the tyrosine. Second, a lysine residue is acetylated. As expected from the characterization of acetylated $\alpha$-tubulin of Chlamydomonas (LeDizet and Piperno, 1987) this residue is Lys 40 and our sequence results show that this position is completely modified in $\alpha$-tubulin from isolated flagella of Trypanosoma.

We have purified the carboxy-terminal fragments of the $\alpha$ and $\beta$-tubulin present in stable microtubules of Trypanosoma. A combined sequence and mass spectrometrical approach provides a catalogue of a rather larger number of tubulin variants, all of which arise from posttranslational modifications (Table 1). We confirm the presence of tyrosinated and detyrosinated $\alpha$-tubulin (for references see above) and show that $\beta$-tubulin displays an unexpected carboxy-terminal processing. More importantly both $\alpha$ - and $\beta$-tubulin are extensively glutamylated while polyglycylation is not detected.

A $\beta$-tubulin sequence ending with a tyrosine (Kimmel et al., 1985 ) is unusual and our results show that trypanosomal $\beta$ tubulin occurs both with and without the tyrosine (Figs 3 and 4; Table 1). Whether this unexpected processing is due to the same enzyme, which acts on $\alpha$-tubulin, or a distinct activity is currently not known. However, the previous tyrosine labelling studies of Trypanosoma cells under conditions of inhibited protein biosynthesis unambiguously show that only detyrosinated $\alpha$-tubulin is again tyrosinated by the tubulin-tyrosine ligase (Sherwin et al., 1987). This also agrees with the substrate sequence requirements of the ligase. At least the mammalian enzyme adds the tyrosine only to a terminal glutamic acid residue (Rüdiger et al., 1994), which is present in Trypanosoma detyrosinated $\alpha$-tubulin while the $\beta$-tubulin ends with sequence QY (Kimmel et al., 1985; Fig. 3).

Polyglutamylation was discovered by mass spectrometry of the carboxy-terminal peptides of mammalian brain $\alpha$-tubulins (Eddé et al., 1990) and subsequently also documented for all different brain $\beta$-tubulins (see Mary et al., 1994 and Rüdiger et al., 1995b, for review). Studies with the monoclonal antibody GT 335, which seems specific for glutamylated tubulins, revealed glutamylation also in non neuronal tissues albeit at a much lower level (Wolff et al., 1992) and in sperm axonemes of mammals (Fouquet et al., 1994), echinoderms (Gagnon et al., 1996) and Drosophila (Bré et al., 1996). GT 335 also revealed glutamylated tubulin in cytoplasmic and axonemal microtubules of Paramecium and other ciliates. However two dimensional immunoblots showed that glutamylated tubulin variants are only a minor component of the ciliary axonemes of Paramecium (Bré et al., 1994), which strongly display polyglycylated $\alpha$ - and $\beta$-tubulins (Redeker et al., 1994).

Our characterization of the carboxy-terminal tubulin fragments by mass spectrometry and sequence analysis gives a quantitative view of the tubulin variants present in a cytoskeletal preparation of Trypanosoma. Both $\alpha$ - and $\beta$ tubulin are glutamylated and the modification involves both tyrosinated and detyrosinated tubulins. The lateral chains range from 1 to 6 residues in $\beta$ while in $\alpha$ the length can reach 15 residues (Table 1 ). The highest glutamylation level (5 to 15 residues) was found in a moderately abundant $\alpha$ variant which 
was detyrosinated. Most surprising is the extent of this posttranslational modification since unglutamylated $\alpha$ - and $\beta$ tubulins are only minor variants in the cytoskeleton, which contains both the subpellicular and the flagellar microtubules. In isolated flagella only glutamylated variants of $\alpha$ - and $\beta$ tubulin were observed. The extent of glutamylation is higher than in preparations of mammalian brain tubulin where unmodified tubulin variants are still present in moderate amounts (Eddé et al., 1990; Mary et al., 1994; Redeker et al., 1992; Rüdiger et al., 1992, 1995b). Our results also provide the first identification of glutamylation sites for a protozoan tubulin. Just as in brain $\alpha$-tubulins (Eddé et al., 1990) the sidechain of Trypanosoma $\alpha$-tubulin is linked to glutamic acid 455 (Fig. 3). The $\beta$-tubulins from brain and Trypanosoma share a glutamylation site at glutamic acid 435 (Fig. 3; Mary et al., 1994; Redeker et al., 1992; Rüdiger et al., 1992).

Mass spectrometry was also instrumental in establishing polyglycylation as a posttranslational modification of $\alpha$ - and $\beta$-tubulin in microtubules of various axonemes. Interestingly polyglycylation is remarkably extensive in cilia of Paramecium (Redeker et al., 1994) while flagella of bull sperm (Rüdiger et al., 1995a; K. Weber and U. Plessman, unpublished results) and sea urchin (Mary et al., 1996; Multigner et al., 1996) show a sizeable fraction of unmodified tubulins. Monoclonal antibodies specific for certain polyglycylated tubulins extend this axonemal location also to sperm of Drosophila and other metazoa (Bré et al., 1996; Bressac et al., 1995). Thus it is surprising that we did not detect polyglycylated tubulin in a cytoskeletal preparation of Trypanosoma to which the single axoneme contributes about $30 \%$ of the total tubulin (Schneider et al., 1987). In addition experiments on the carboxy-terminal fragments of $\alpha$ - and $\beta$ tubulin from purified flagella showed again only glutamylated tubulins with and without the terminal tyrosine. Furthermore Trypanosoma cells put under conditions of strict inhibition of protein biosynthesis incorporate into tubulin not only radioactively labelled acetate and tyrosine (Schneider et al., 1987; Sherwin et al., 1987) but also glutamate while no incorporation of glycine was observed (Fig. 5). Thus the amount of glycylated variants is either very small and remained undetected or alternatively these variants may not arise in axonemes of Trypanosoma. This may also explain why none of the various antibodies to polyglycylated tubulins reacted on Trypanosoma (Bré et al., 1996).

The absence of polyglycylation in Trypanosoma axonemes is probably not due to evolutionary considerations. Although Kinetoplastida are relatively primitive eukaryotes (Sogin et al., 1989) polyglycylation occurs already in the diplomonad Giardia lamblia (Weber et al., 1996) which is usually considered to be one of the oldest eukaryotes (Sogin et al., 1989; Leipe et al., 1993). About half the $\alpha$-tubulin of Giardia, which has 8 normal axonemes, is heavily glycylated with lateral chains between 2 and 23 glycyl residues in length. We also do not know whether lack of polyglycylation somehow relates to the peculiarity that a unique structure, the paraflagellar rod, is present next to the trypanosomal axoneme. An alternative speculation relates to the remarkably high polyglutamylation level of all microtubules in Trypanosoma (see Results) which may be related to a particularly high level of glutamylation enzymes. Since polyglutamylation and polyglycylation involve very closely spaced glutamyl residues or potentially even the same residue, glycylation may not be possible in fully glutamylated microtubules.

Tubulin modification by polyglutamylation and polyglycylation seem widely spread in eukaryotes, but their function remains a matter of debate. Recently Boucher et al. (1994) reported that the length of the oligoglutamyl sidechain in brain tubulin modulates the affinity for the microtubule associated protein tau through a progressive conformational shift of the carboxy-terminal domain of tubulin. The relative affinity for tau increases with one to three glutamyl units, reaches an optimum and decreases progressively when the sidechain lengthens up to six residues. Similar observations seem to hold for MAP 2 (Boucher et al., 1994). Thus one wonders whether polyglutamylation can also modulate the interaction with microtubule associated proteins in trypanosomes. Their subpellicular microtubules are unusually stable and morphological and biochemical studies document a wealth of microtubule associated proteins. Their abundance may suggest that the unusual stability of the microtubules arises from the extensive crosslinking between the microtubules and between the cell membrane and the microtubules (reviewed by Seebeck et al., 1990). If posttranslational modifications accumulate preferentially on polymeric tubulin the extensive glutamylation observed in this study could give rise to a particularly stable population of microtubules via an enhanced binding of some associated proteins. Some aspects of this speculation can be tested in the future. In addition Trypanosoma may be a particularly good system to address polyglutamylation by the future purification of the enzyme(s) involved in this process. Furthermore trypanosomes are amenable to genetic techniques such as gene replacements which may provide a novel approach to functional studies.

This work was supported by grant 31-46628-96 from the Swiss National Foundation (to A.S.), by a fellowship of the 'Prof. Dr Max Cloëtta' - Foundation (to A.S.) and by funds of the Max Planck Society (to K.W.). We thank Elke Horn for excellent technical assistance.

\section{REFERENCES}

Alexander, J. E., Hunt, D. F., Lee, M. K., Shabanowitz, J., Michel, H., Berlin, S. C., MacDonald, T. L., Sundberg, R. J., Rebhuhn, L. and Frankfurter, A. (1991). Characterization of posttranslational modifications in neuron-specific class III $\beta$ tubulin by mass spectrometry. Proc. Nat. Acad. Sci. USA 88, 4685-4689.

Boucher, D., Larcher, J.-C., Gros, F. and Denoulet, P. (1994). Polyglutamylation of tubulin as a progressive regulator of in vitro interactions between the microtubule-associated protein tau and tubulin. Biochemistry 33, 12471-12477.

Bré, M. H., de Néchaud, B., Wolff, A. and Fleury, A. (1994). Glutamylated tubulin probed in ciliates with the monoclonal antibody GT 335. Cell Mot. Cytoskeleton 27, 337-349.

Bré, M.-H., Redeker, V., Quibell, M., Darmanaden-Delorme, J., Bressac, C., Cosson, J., Huitorel, P., Schmitter, J.-M., Rossier, J., Johnson, T., Adoutte, A. and Levilliers, N. (1996). Axonemal tubulin polyglycylation probed with two monoclonal antibodies: widespread evolutionary distribution, appearance during spermatozoan maturation and possible function in motility. J. Cell Sci. 109, 727-738.

Bressac, C., Bré, M.-H., Darmanaden-Delorme, J., Laurent, M., Levilliers, N. and Fleury, A. (1995). A massive new posttranslational modification occurs on axonemal tubulin at the final step of spermatogenesis in Drosophila. Eur. J. Cell Biol. 67, 346-355.

Cavalier-Smith, T. (1993). The kingdom Protista and its 18 phyla. Microbiol. Rev. 57, 953-994. 
Eddé, B., Rossier, J., Le Caer, J.-P., Desbruyères, E., Gros, F. and Denoulet, P. (1990). Posttranslational glutamylation of $\alpha$-tubulin. Science 247, 83-84.

Ersfeld, K., Wehland, J., Plessmann, U., Dodemont, H., Gerke, V. and Weber, K. (1993). Characterization of the tubulin-tyrosine ligase. J. Cell Biol. 120, 725-732.

Fouquet, J.-P., Eddé, B., Kann, M.-L., Wolff, A., Desbuyères, E., Gros, F. and Denoulet, P. (1994). Differential distribution of glutamylated tubulin during spermatogenesis in mammalian tissues. Cell Motil. Cytoskel. 27, 4958.

Gagnon, C., White, D., Cosson, J., Huitorel, P., Eddé, B., Desbruyères, E., Paturle-Lafanechère, L., Multigner, L., Job, D. and Cibert, C. (1996). The polyglutamylated lateral chain of alpha-tubulin plays a key role in flagellar motility. J. Cell Sci. 109, 1545-1553.

Kimmel, B. E., Samson, S., Wu, J., Hirschberg, R. and Yarbrough, L. R. (1985). Tubulin genes of the African trypanosome Trypanosoma brucei rhodesiense sequence of a $3.7 \mathrm{~Kb}$ fragment containing genes for $\alpha$ - and $\beta$ tubulins. Gene 35, 237-248.

LeDizet, M. and Piperno, G. (1987). Identification of an acetylation site of Chlamydomonas $\alpha$-tubulin. Proc. Nat. Acad. Sci. USA 84, 5720-5724.

Leipe, D. D., Gunderson, J. H., Nerad, T. A. and Sogin, M. L. (1993). Small subunit ribosomal $\mathrm{RNA}^{+}$of Hexamita inflata and the quest for the first branch in the eukaryotic tree. Mol. Biochem. Parasitol. 59, 41-48.

Mary, J., Redeker, V., Le Caer, J.-P., Promé, J.-C. and Rossier, J. (1994). Class I and IVa $\beta$ tubulin isotypes expressed in adult mouse brain are glutamylated. FEBS Lett. 353, 89-94.

Mary, J., Redeker, V., Le Caer, J.-P., Rossier, J. and Schmitter, J.-M. (1996). Posttranslational modifications in the C-terminal tail of axonemal tubulin from sea urchin sperm. J. Biol. Chem. 271, 9928-9933.

Multigner, L., Pignot-Paintrand, I., Saoudi, Y., Job, D., Plessmann, U., Rüdiger, M. and Weber, K. (1996). The A and B tubules of the outer doublets of sea urchin sperm axonemes are composed of different tubulin variants. Biochemistry 33, 10862-10871.

Paturle, L., Wehland, J., Margolis, R. L. and Job, D. (1989). Complete separation of tyrosinated, detyrosinated, and nontyrosinatable brain tubulin subpopulations using affinity chromatography. Biochemistry 28, 2698-2704.

Paturle-Lafanechère, L., Eddé, B., Denoulet, P., Van Dorsselaer, A., Mazarguil, H., Le Caer, J.-P., Wehland, J. and Job, D. (1991). Characterization of a major brain tubulin variant which cannot be tyrosinated. Biochemistry 30, 10523-10528.

Raybin, D. and Flavin, M. (1975). An enzyme tyrosylating $\alpha$ tubulin and its role in microtubule assembly. Biochem. Biophys. Res. Commun. 65, 10881095.

Redeker, V., Melki, R., Promé, D., Le Caer, J.-P. and Rossier, J. (1992). Structure of tubulin C-terminal domain obtained by subtilisin treatment. FEBS Lett. 313, 185-192.

Redeker, V., Levilliers, N., Schmitter, J.-M., Le Caer, J.-P., Rossier, J., Adoutte, A. and Bré, M.-H. (1994). Polyglycylation of tubulin: a posttranslational modification in axonemal microtubules. Science 266, 16881691.

Rider, M. H., Puype, M., Van Damme, J., Gevaert, K., De Roeck, S., D'Alayer, J., Rasmussen, H. H., Celis, J. E. and Vandeckerkhove, J. (1995). An agarose-based gel-concentration system for microsequence and mass spectrometric characterization of proteins previously purified in polyacrylamide gels starting at low picomole levels. Eur. J. Biochem. 230, 258-265.
Rüdiger, M., Plessmann, U., Klöppel, K.-D., Wehland, J. and Weber, K. (1992). Class II tubulin, the major brain $\beta$ tubulin isotype is polyglutamylated on glutamic acid residue 435. FEBS Lett. 308, 101-105.

Rüdiger, M. and Weber, K. (1993). Characterization of the posttranslational modifications in tubulin from the marginal band of avian erythrocytes. Eur. $J$. Biochem. 218, 107-116.

Rüdiger, M., Wehland, J. and Weber, K. (1994). The carboxy-terminal peptide of detyrosinated $\alpha$ tubulin provides a minimal system to study the substrate specificity of tubulin-tyrosine ligase. Eur. J. Biochem. 220, 309320.

Rüdiger, M., Plessmann, U., Rüdiger, A.-H. and Weber, K. (1995a). $\beta$ Tubulin of bull sperm is polyglycylated. FEBS Lett. 364, 147-151.

Rüdiger, A., Rüdiger, M., Weber, K. and Schomburg, D. (1995b). Characterization of post-translational modifications of brain tubulin by matrix-assisted laser desorption/ionization mass spectroscopy: direct onestep analysis of a limited subtilisin digest. Anal. Biochem. 224, 532-537.

Schlaeppi, K., Deflorin, J. and Seebeck, T. (1989). The major component of the paraflagellar rod of Trypanosoma brucei is a helical protein that is encoded by two identical, tandemly linked genes. J. Cell Biol. 109, 16951709.

Schneider, A., Sherwin, T., Sasse, R., Russell, D. G., Gull, K. and Seebeck, T. (1987). Subpellicular and flagellar microtubules of Trypanosoma brucei brucei contain the same $\alpha$-tubulin isoforms. J. Cell Biol. 104, 431-438.

Seebeck, T. and Kurath, U. (1985). Two simple media for biochemical experimentation with cultured procyclic Trypanosoma brucei. Acta Tropica 42, 127-136.

Seebeck, T., Schneider, A., Kueng, V., Schlaeppi, K. and Hemphill, A. (1988). The cytoskeleton of Trypanosoma brucei - the beauty of simplicity. Protoplasma 145, 188-194.

Seebeck, T., Hemphill, A. and Lawson, D. (1990). The cytoskeleton of trypanosomes. Parasitol. Today 6, 49-52.

Sherwin, T., Schneider, A., Sasse, R., Seebeck, T. and Gull, K. (1987). Distinct localization and cell cycle dependence of $\mathrm{COOH}$ terminally tyrosinolated $\alpha$-tubulin in the microtubules of Trypanosoma brucei brucei. J. Cell Biol. 104, 439-446.

Sogin, M. L., Gunderson, J. H., Elwood, H. J., Alonso, R. A. and Peattie, D. A. (1989). Phylogenetic meaning of the kingdom concept: an unusual ribosomal RNA from Giardia lamblia. Science 243, 75-77.

Stieger, J., Wyler, T. and Seebeck, T. (1984). Partial purification and characterization of microtubular protein from Trypanosoma brucei. J. Biol. Chem. 259, 4596-4602.

Thompson, W. C. (1982). The cyclic tyrosination/detyrosination of alpha tubulin. Meth. Cell. Biol. 24, 235-255.

Weber, K., Schneider, A., Müller, N. and Plessmann, U. (1996). Polyglycylation of tubulin in the diplomonad Giardia lamblia, one of the oldest eukaryotes. FEBS Lett. 393, 27-30.

Wolff, A., de Néchaud, B., Chillet, D., Mazarguil, H., Desbruyères, E., Audebert, S., Eddé, B., Gros, F. and Denoulet, P. (1992). Distribution of glutamylated $\alpha$ - and $\beta$-tubulin in mouse tissues using a specific monoclonal antibody, GT335. Eur. J. Cell Biol. 59, 425-432.

(Received 15 November 1996 - Accepted 20 December 1996) 